\title{
Video Article \\ Sorting of Streptomyces Cell Pellets Using a Complex Object Parametric Analyzer and Sorter
}

\author{
Marloes L. C. Petrus ${ }^{1}$, G. Jerre van Veluw ${ }^{2}$, Han A. B. Wösten ${ }^{2}$, Dennis Claessen ${ }^{1}$ \\ ${ }^{1}$ Microbial Biotechnology, Institute Biology Leiden, Leiden University \\ ${ }^{2}$ Microbiology, Kluyver Centre for Genomics of Industrial Fermentation, Utrecht University
}

Correspondence to: Dennis Claessen at d.claessen@biology.leidenuniv.nl

URL: https://www.jove.com/video/51178

DOI: doi: $10.3791 / 51178$

Keywords: Microbiology, Issue 84, Streptomyces, flow cytometry, pellets, morphology, fluorescence, COPAS, biotechnology, high-throughput analysis, sorting, heterogeneity

Date Published: 2/13/2014

Citation: Petrus, M.L., van Veluw, G.J., Wösten, H.A., Claessen, D. Sorting of Streptomyces Cell Pellets Using a Complex Object Parametric Analyzer and Sorter. J. Vis. Exp. (84), e51178, doi:10.3791/51178 (2014).

\section{Abstract}

Streptomycetes are filamentous soil bacteria that are used in industry for the production of enzymes and antibiotics. When grown in bioreactors, these organisms form networks of interconnected hyphae, known as pellets, which are heterogeneous in size. Here we describe a method to analyze and sort mycelial pellets using a Complex Object Parametric Analyzer and Sorter (COPAS). Detailed instructions are given for the use of the instrument and the basic statistical analysis of the data. We furthermore describe how pellets can be sorted according to user-defined settings, which enables downstream processing such as the analysis of the RNA or protein content. Using this methodology the mechanism underlying heterogeneous growth can be tackled. This will be instrumental for improving streptomycetes as a cell factory, considering the fact that productivity correlates with pellet size.

\section{Video Link}

The video component of this article can be found at https://www.jove.com/video/51178/

\section{Introduction}

Streptomycetes are filamentous soil bacteria that are well known for their proficiency to make antibiotics, as well as compounds that can be used as immunosuppressants or to combat fungal infections or cancer ${ }^{1,2}$. In addition, these organisms produce enzymes that are of interest for a wide range of industrial applications ${ }^{3}$. Most of these commercially interesting compounds are produced in bioreactors. Growth of streptomycetes in bioreactors is characterized by the formation of complex structures of interconnected hyphae, known as clumps or pellets. These multicellular structures are highly heterogeneous with respect to size ${ }^{4}$ and can reach sizes that are more than a million times larger than a single-celled bacterium such as Escherichia coli or Bacillus subtilis. Although heterogeneity is regarded as a beneficial trait in natural biological systems ${ }^{5}$, it is considered a production pitfall in industry. Bioreactor cultivations have to be reproducible and controllable to obtain the highest possible yields. A detailed understanding of the role of each of the pellet types in a bioreactor is therefore crucial to improve streptomycetes as a cell factory.

Flow cytometry is commonly used to analyze individual cells in a population ${ }^{6}$. Flow cytometers can acquire multiparametric information by simultaneously measuring characteristics of the cells (such as size, density, and multicolor fluorescence). In this way, cell properties can be correlated thereby contributing to our understanding of heterogeneity within a culture and the existence of distinct populations of cells ${ }^{6}$. More specialized instruments have made it possible to sort cells according to user-defined parameters. For instance, mutants can be screened. Following sorting, such mutant cells can be cultivated for further characterization. This has already proven to be useful, amongst others, to improve productivity of strains $s^{7,8}$. The nozzles of flow cytometers typically allow for the passage of cells with a maximal diameter of approximately $10 \mu \mathrm{m}$. Therefore, pellets of streptomycetes cannot be analyzed with regular flow cytometers. They, however, can be analyzed with the Complex Object Parametric Analyzer and Sorter (COPAS). Like regular flow cytometers, COPAS can acquire multiparametric data of particles in a high throughput manner. Depending on the type of COPAS 10-1,500 $\mu \mathrm{m}$ sized particles can be analyzed. In addition, it allows for sorting of individual particles that can be used for cultivation or downstream analyses, such as the isolation of DNA, RNA, or proteins. COPAS was initially designed for the analysis and sorting of small multicellular organisms, such as the nematode Caenorhabditis elegans ${ }^{9}$, and Drosophila embryos and larvae $^{10}$. The instruments have also been used for zebrafish ${ }^{11}$ and for filamentous fungi ${ }^{12,13}$. The latter organisms also form mycelial pellets that are even larger than those formed by filamentous bacteria. We have recently demonstrated that the use of COPAS is also feasible for streptomycetes ${ }^{4}$. We here describe the experimental procedure for using the COPAS to assess pellet heterogeneity in Streptomyces coelicolor, including details on the methodology to sort pellets according to size. Please note, however, that this method can also be used for the analysis of other pellet-forming streptomycetes. 


\section{Protocol}

The procedure to analyze and sort Streptomyces pellets from a two-day-old liquid-grown culture is schematically represented in Figure 1. Details for the method are given below.

\section{Growth (Including Preparation of Media and Buffers)}

1. Prepare $1 \mathrm{~L}$ of $10 x$ phosphate buffered saline (PBS; $80 \mathrm{~g} \mathrm{NaCl}, 2 \mathrm{~g} \mathrm{KCl}, 14.4 \mathrm{~g} \mathrm{Na}_{2} \mathrm{HPO}_{4}, 2.4 \mathrm{~g} \mathrm{KH}_{2} \mathrm{PO}_{4}$ in $1 \mathrm{~L}$ of distilled water adjusted to $\mathrm{pH}$ 7.4). At time of use add $100 \mathrm{ml}$ of $10 x$ PBS to $900 \mathrm{ml}$ of distilled water to obtain $1 \mathrm{~L}$ of PBS.

2. Prepare $1 \mathrm{~L}$ of YEME medium (3 g Difco yeast extract, $5 \mathrm{~g}$ Bacto peptone, $3 \mathrm{~g}$ Oxoid malt extract, $10 \mathrm{~g}$ glucose, $340 \mathrm{~g}$ sucrose, distilled water up to $1 \mathrm{~L}$ ) and $100 \mathrm{ml}$ of $2.5 \mathrm{M} \mathrm{MgCl}_{2}$. Sterilize both solutions by autoclaving. Note that also other Streptomyces media can be used ${ }^{4,14}$.

3. Add $100 \mathrm{ml}$ YEME medium and $0.2 \mathrm{ml} 2.5 \mathrm{M} \mathrm{MgCl}_{2}$ to a sterile $250 \mathrm{ml}$ Erlenmeyer flask equipped with metal coiled springs. Prepare as many flasks as bacterial samples to study.

4. Inoculate each flask with $10^{8}$ spores of Streptomyces to obtain a spore concentration of $10^{6}$ spores $/ \mathrm{ml}$. Grow the bacteria for 2 days at $30{ }^{\circ} \mathrm{C}$ while shaking at $180 \mathrm{rpm}$.

\section{Sampling}

1. Transfer a $5 \mathrm{ml}$ sample from each culture to a $15 \mathrm{ml}$ Falcon tube using a sterile $5 \mathrm{ml}$ pipette. Gently shake the culture while taking the sample to ensure that the sample is representative for the whole culture.

2. When samples are analyzed immediately with COPAS no sample preparation steps are necessary. Keep the sample on ice and proceed to step 3.1 of this protocol. When samples are analyzed at a later time point, fix the pellets as described in steps $2.3-2.5$ of this protocol. Note that fixation also prevents growth of the bacteria in the tubing system when not properly cleaned after analyses. Ensure that fixation with formaldehyde is not hampering downstream analyses. For instance, RNA could not be isolated from fungal pellets after fixation with formaldehyde. In contrast, RNA was successfully isolated after fixation with $70 \%$ ethanol ${ }^{12}$.

3. Add $600 \mu \mathrm{l}$ of $37 \%$ formaldehyde to $5 \mathrm{ml}$ of sample and mix by inverting the tube $3 \mathrm{x}$. Fix the samples on ice for $30 \mathrm{~min}$.

4. Centrifuge the samples at $2,500 \times \mathrm{g}$ in a fixed rotor at $4{ }^{\circ} \mathrm{C}$ for $10 \mathrm{~min}$. Carefully remove the supernatant and wash the pellets with $5 \mathrm{ml}$ of PBS.

5. Repeat step 2.4 so that the pellets are washed with PBS $2 x$. The samples can be stored in PBS at $4{ }^{\circ} \mathrm{C}$ for several weeks.

\section{COPAS Analysis}

1. Make sure the COPAS Plus, the pump, the computer and the $488 \mathrm{~nm}$ argon laser are turned on and start the Biosort software. Although turned on, the laser will still be in standby modus.

2. Check if the sheath fluid bottle is full and the waste bottle is empty.

3. Click 'START' and then 'RUN' to switch the $488 \mathrm{~nm}$ argon laser from standby to on. After approximately 60 sec, when the laser is on, click 'DONE'.

4. Check the pressure gauges. The pressures for 'Sheath', 'Sample', 'Sorter' and 'Clean' should read around 4.0, 0.5, 2.7, and 10.9 respectively.

5. Click the checkbox next to 'Pressure OK'. The system then primes the flow cell and is ready for use.

6. Standard settings are assumed with 'Delay' at 11 and 'Width' 7 . Thresholds are set at 50 for 'Signal' and 40 for 'TOF Minimum', with TOF representing the time-of-flight. (For completeness: all 'Gains' are set at 1, the trigger is set at EXT (Extinction) and in the 'Configuration' tab under 'Select scan rate' $2.50 \mathrm{MHz}$ is chosen. Coincidence is set to 'Enhanced').

7. Remove leftover water from the sample cup and add $0.1 \mathrm{ml}$ of Streptomyces sample from step 2.2 or 2.5 in the cup together with PBS (around $50 \mathrm{ml}$ ). Make sure the sample cup is closed properly.

8. Click 'Acquire' to start collecting data. Manage the flow speed to obtain between 30-50 events per second. If the flow is too high, add PBS to the sample cup. If the flow speed is too low, add more sample.

9. When at least 2,500 events are collected click 'STOP'. To save all data click 'Store' and save your file for subsequent statistical analysis. The Biosort software will save the data in four files, of which only the .txt file will be used for the subsequent analysis.

10. Clean the sample cup by removing the sample with a $50 \mathrm{ml}$ syringe and washing with water $2 \mathrm{x}$.

11. Repeat steps 3.7-3.10 until all samples are analyzed.

\section{Data Analysis}

1. Open the .txt file and discard data with an EXT lower than 25 (using for instance MS Excel). This corresponds to debris and loose hyphal fragments (for Aspergillus niger all data with an EXT lower than 150 are discarded) ${ }^{4,12}$. Then, save all TOFs in one column in a plain '.txt' file (for many data files this can be automated in the programming language R, available from: http://www.r-project.org).

2. Make sure SciLab (version 5.3.2 or newer from http://www.scilab.org/) is installed together with the 'fittools' library, which is available, together with the manual, from: http://web.science.uu.nl/microbiology/images/fung/fittools.zip http://web.science.uu.nl/microbiology/images/fung/manual\%20fittools.pdf

3. Start SciLab and run the necessary functions of the fittools library to model the data. In short: Install the fittools library as shown in the manual (only needed once).

1. Load the fittools library.

2. Read the data ('.txt' file) into SciLab.

3. Log transform the data (natural log is the default log function). 
4. Fit the model of 2 populations to the data.

5. Plot a graph with the histogram and model (optional).

6. Bootstrap the data (1,000 replicates).

7. Fit the model to the bootstrapped datasets.

8. Get the confidence intervals estimates of the 5 parameters. This will provide information on the participation fraction $(p)$ of the populations, their 2 means $\left(\mu_{1}\right.$ and $\left.\mu_{2}\right)$ and the accompanying standard deviations $\left(\alpha_{1}\right.$ and $\left.\alpha_{2}\right)$. Furthermore, the $95 \%$ confidence intervals are determined by refitting with the model after bootstrapping $(1,000 \text { replicates })^{12,13,15}$. When the confidence intervals of the means do not overlap and the confidence interval of $p$ is between $0.025-0.975$ the dataset can be explained as a combination of two populations of normal distributions. (Note that the participation fraction of small pellets is $p$, whereas the participation of large pellets is 1-p.) The relationship between TOF and the diameter of a Streptomyces pellet equals $0.57 \times \mathrm{TOF}+159 \mu \mathrm{m}^{4}$.

4. In the case two populations are found use the average TOFs as sorting parameters in the COPAS as the upper and lower bound to sort the small and large pellets subsequently. Note that also other sorting parameters can be selected depending on the demands of the user.

\section{Pellet Sorting}

1. Load the Streptomyces sample that needs to be sorted as described in step 3.7.

2. Set 'Sorting Limits' and provide details about the minimal (Lo) and maximal (Hi) TOF values. Alternatively, set an area by selecting 'Regions' and then 'Define Gate Region' to select the pellets with the desired dimensions or properties.

3. Place a $50 \mathrm{ml}$ tube to collect multiple pellets that meet the sorting parameters. Alternatively, a micro-titer plate can be used to sort individual pellets. Multiple pellets $\left(10^{4}-10^{5}\right)$ are needed for DNA and protein extraction. A single pellet is sufficient for qPCR analysis of gene expression, but multiple pellets are needed for RNA sequencing.

4. Decide and set the number of pellets that need to be sorted and click 'Sort Manually' to sort for the selected parameters. When the set amount of pellets is reached, the sorting ends automatically.

5. Remove left over sample with a $50 \mathrm{ml}$ syringe from the sample cup and wash the cup with water $2 \mathrm{x}$. Repeat steps 5.1-5.5 if other samples need to be sorted.

6. Before shutting down the COPAS rinse the sample cup with water to remove the remaining pellets and clean the sample cup with $70 \%$ EtOH and/or $2 \%$ hypochlorite in water. Run a sample of chlorinated water to clean the entire system and repeat this with water. Empty the overflow container and clean or replace the filter that resides in this container.

7. When finished, click 'STOP' to release the pressure from the system. When the motor stops close the program and click 'Shutdown without purging'. Then switch off the computer, the COPAS, the laser, and the pump.

\section{Representative Results}

\section{COPAS measurements of Streptomyces pellets}

Streptomycetes form mycelial pellets in liquid cultures that have a wide range of sizes. To analyze pellet size distribution, a 2-day old liquid grown Streptomyces coelicolor culture was subjected to large particle flow cytometry using a COPAS Plus Profiler equipped with a $1 \mathrm{~mm}$ nozzle. A typical COPAS output is a scatter plot such as visualized in Figure 2A. The x-axis represents the time-of-flight (TOF), which correlates with pellet size (i.e. it takes longer for larger pellets to pass the laser beam). The $y$-axis shows the extinction, which represents the optical density of the object. Each point in the scatter plot corresponds to an individual event, i.e. a single pellet passing the laser beam. Importantly, when the sample is too concentrated (i.e. when the laser detects more than 100 events/sec), the COPAS frequently fails to detect individual pellets. This leads to false TOF values that are too high. Diluting the sample reduces the average TOF values, up to a point where further diluting does no longer impact TOF. This point is reached when approximately 100 pellets/sec were analyzed.

Plotting the data points into a histogram indicates that the sizes are not normally distributed (Figure 2B). The distribution appears to be skewed to the right. Log transforming the data set also did not lead to a normal distribution (Figure 2C). To assess whether the size distribution can be explained by assuming a mixture of two normal distributions the data was mathematically modeled ${ }^{12,15}$. Modeling indeed indicated that the size distribution can be explained by assuming the existence of two distinct populations of pellets. The population of small pellets consisted of $92 \%$ of all pellets with an average size of $248 \mu \mathrm{m}$, while the population of large pellets ( $8 \%$ of all pellets) had an average size of $319 \mu \mathrm{m}$ (note that two populations are assumed to exist when the participation fraction is between $2.5-97.5 \%$ ).

Sorting of Streptomyces pellets according to size

Micro-colonies from the populations of large and small pellets were sorted. To this end, the average pellet sizes of the two populations were used to define the boundaries for sorting. Pellets smaller than $248 \mu \mathrm{m}$ were considered to be from the small pellet population, whereas pellets larger than $319 \mu \mathrm{m}$ were considered to be from the large pellet population (Figure 3), so as to limit sorting of pellets from the overlapping portion of the two size distributions. Microscopic analysis of sorted pellets showed their distinct sizes (Figure 3). The collected pellets can be further used for DNA, RNA, or protein isolations. 


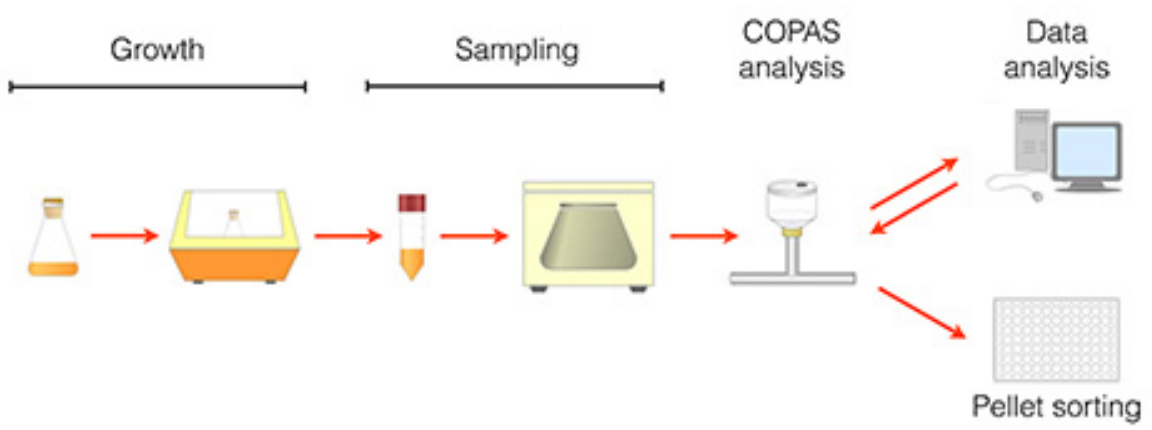

Figure 1. Schematic representation of the experimental setup to measure and analyze Streptomyces pellets using COPAS. For details, see experimental procedures. Click here to view larger image.

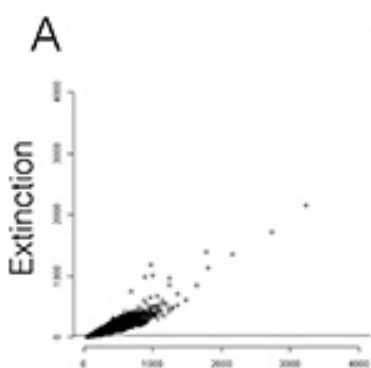

Time of Flight

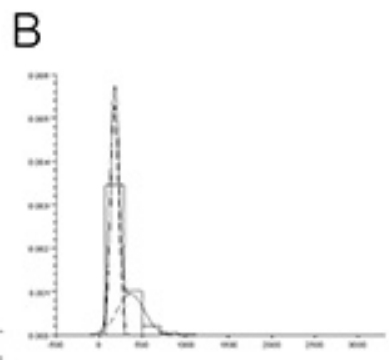

Time of Flight

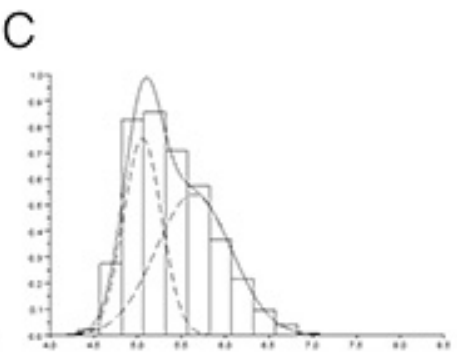

LOG Time of Flight

Figure 2. Pellet size heterogeneity in liquid-grown Streptomyces cultures. COPAS analysis of a 2-day-old S. coelicolor YEME culture (A) indicates that pellet sizes (indicated as Time of Flight values) are not normally distributed (B), and neither become so when log-transformed (C). Instead, two populations of pellets that differ in TOF (and thus size) were detected. Click here to view larger image.

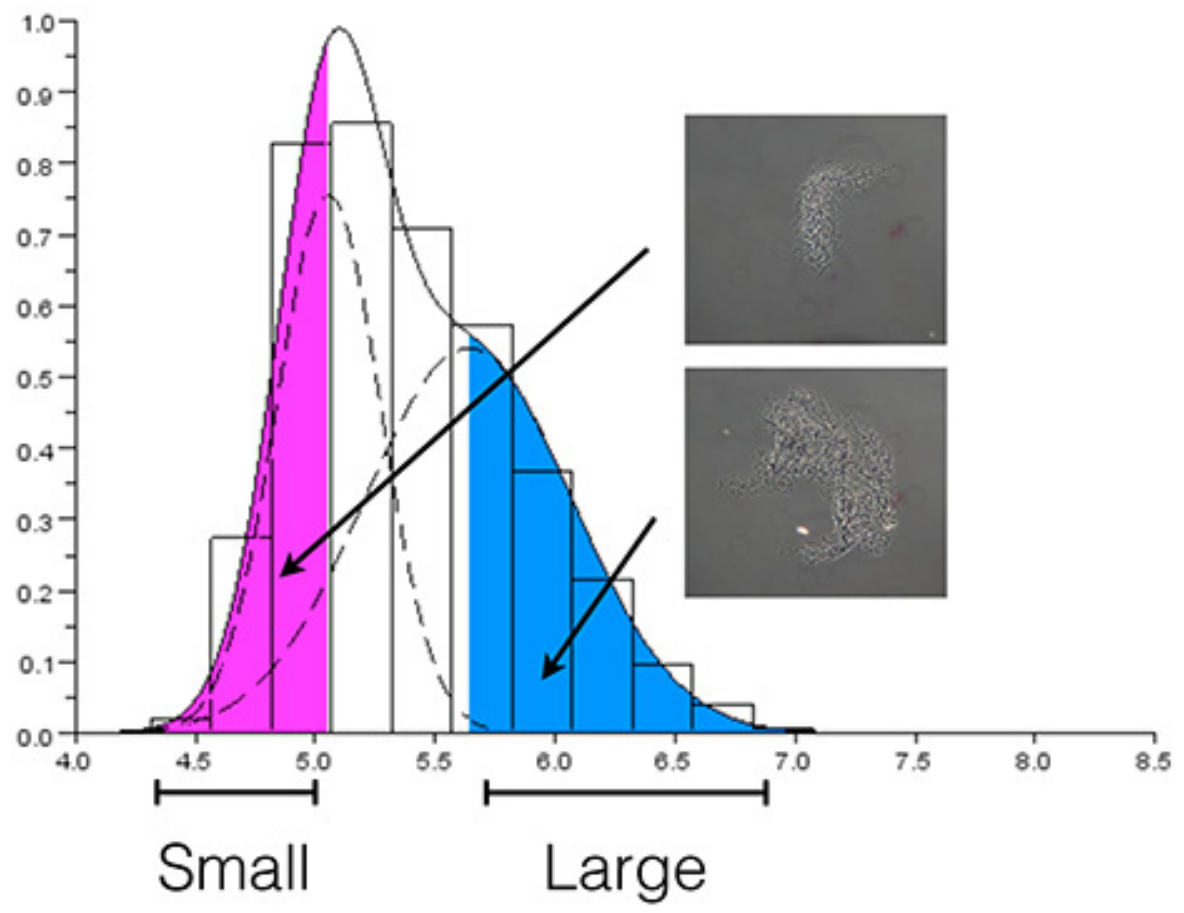

Figure 3. Fractionation of Streptomyces pellets according to size. Pellets with a size smaller than $248 \mu \mathrm{m}$ (indicated in pink) were considered to be small pellets, whereas pellets with a size larger than $319 \mu \mathrm{m}$ (indicated in blue) were considered to be large pellets. Microscopic analysis confirmed the difference in size. Note that these sizes were calculated from the log-transformed data using the described relationship between TOF and the diameter of a Streptomyces pellet, which equals $0.57 \times$ TOF $+159 \mu \mathrm{m}$. Click here to view larger image. 


\section{Discussion}

Flow cytometry has enabled the high-speed analysis of large numbers of single cells, which contributed to our understanding of heterogeneity in clonal populations ${ }^{6}$. Regular flow cytometry is not feasible for analysis of the multicellular mycelial pellets of streptomycetes and fungi. Our work has demonstrated that high-throughput analysis of Streptomyces pellets is feasible using COPAS. The procedure outlined here is simple, fast, and highly reproducible. The critical parameter to keep in mind during operation of the instrument is the flow speed, which should not exceed 100 events/sec (step 3.8 of this protocol). If the pellet concentration, and therefore also the flow speed, becomes too high, the TOF values will be miscalculated because the instrument fails to detect individual pellets. Sufficiently diluting the sample by the addition of PBS overcomes this problem.

\section{Limitations}

The COPAS Plus used here has a nozzle diameter of $1 \mathrm{~mm}$, which is suitable for measuring particles with a size ranging from $30-700 \mu \mathrm{m}$. This nozzle therefore enables measuring pellets formed by streptomycetes. In the case of filamentous fungi the micro-colonies may be larger, which limits the general applicability of the COPAS Plus. The COPAS XL can measure particles up to 1,500 $\mu \mathrm{m}$ in size but its sensitivity in the lower range of diameter is less compared to that of the COPAS Plus. Both the COPAS Plus and the COPAS XL cannot analyze particles smaller than $30 \mu \mathrm{m}$. This implies that individual microbial spores or cells cannot be analyzed. In addition, the COPAS may not accurately analyze small aggregates of spores and cells or the very small micro-colonies. For this, regular cell analyzers should be used. This limitation is overcome by the Biosorter of Union Biometrica, which can analyze particles in the range of 1-1,500 $\mu \mathrm{m}$. The purchase prize however is higher.

\section{Troubleshooting}

The COPAS is a robust instrument that is easy to operate. However, sometimes pellets are not detected after loading a sample into the sample cup and starting the measurement. The cause is typically a clogged entry tube, which can easily be solved by pressing the 'clean' command. This will force the pellets back from the tubing system into the sample cup. An alternative reason might be that the lid is not properly placed on the sample cup. This leads to pressure loss and the concomitant failure to detect pellets.

\section{Significance and future directions}

We here focused on the analysis of pellet size but the COPAS setup is also able to analyze and sort based on fluorescence and density. Fluorescence detection enables us to analyze gene expression based on reporters such as GFP. Furthermore, the composition of cells can be evaluated. Even more powerful is the option to separate pellets according to these parameters. Sorted pellets can be used for downstream analyses, including all -omics studies. Indeed, we previously sorted 60,000 large and 200,000 small pellets, and demonstrated that the proteome was significantly different between large and small pellets ${ }^{4}$. This technology therefore provides new leads to improve streptomycetes as cell factories.

The key benefit of the COPAS technology is time. Previous studies on cell pellets were performed using microscopy, and this limits the number of pellets that can be analyzed up to several hundreds ${ }^{16}$. Microscopy studies already suggested the existence of two populations of pellets in liquidgrown Streptomyces cultures ${ }^{16}$. Indeed, two populations of pellets were detected regardless of culture conditions in a large number of different streptomycetes ${ }^{4}$. This size heterogeneity is not restricted to filamentous streptomycetes, but was also observed in filamentous fungi ${ }^{12}$. In all cases, the underlying mechanisms of heterogeneity are not yet known. The possibility to sort pellets according to size and fluorescence enables us to unravel such mechanisms.

\section{Disclosures}

The authors declare that they have no competing financial interests.

\section{References}

1. Hopwood, D.A. Streptomyces in nature and medicine: the antibiotic makers. New York, Oxford University Press (2007).

2. van Wezel, G.P. \& McDowall, K.J. The regulation of the secondary metabolism of Streptomyces: new links and experimental advances. Nat. Prod. Rep. 28, 1311-1333 (2011).

3. Anné, J., Maldonado, B., Van Impe, J., Van Mellaert, L. \& Bernaerts, K. Recombinant protein production and streptomycetes. J. Biotechnol. 158, 159-167 (2012).

4. van Veluw, G.J. et al. Analysis of two distinct mycelial populations in liquid-grown Streptomyces cultures using a flow cytometry-based proteomics approach. Appl. Microbiol. Biotechnol. 96, 1301-1312 (2012).

5. Veening, J.W., Smits, W.K. \& Kuipers, O.P. Bistability, epigenetics, and bet-hedging in bacteria. Annu. Rev. Microbiol. 62, 193-210 (2008).

6. Davey, H.M. \& Winson, M.K. Using flow cytometry to quantify microbial heterogeneity. Curr. Issues Mol. Biol. 5, 9-15 (2003).

7. Betz, J.W., Aretz, W. \& Härtel, W. Use of flow cytometry in industrial microbiology for strain improvement programs. Cytometry. 5, 145-150 (1984).

8. Bell, P. J. et al. A flow cytometric method for rapid selection of novel industrial yeast hybrids. Appl. Environ. Microbiol. 64, 1669-1672 (1998).

9. Pulak, R. Techniques for analysis, sorting, and dispensing of C. elegans on the COPAS flow-sorting system. In Strange, K., Ed., C. elegans: methods and applications. Springer, 275-286 (2006).

10. Buszczak, M. et al. The carnegie protein trap library: a versatile tool for Drosophila developmental studies. Genetics. 175, 1505-1531 (2007).

11. Carvalho, R. et al. A high-throughput screen for tuberculosis progression. PLoS One. 6, e16779 (2011).

12. de Bekker, C., van Veluw, G.J., Vinck, A., Wiebenga, L.A. \& Wösten, H.A.B. Heterogeneity of Aspergillus niger microcolonies in liquid shaken cultures. Appl. Environ. Microbiol. 77, 1263-1267 (2011).

13. van Veluw, G.J. et al. Heterogeneity in liquid shaken cultures of Aspergillus niger inoculated with melanised conidia or conidia of pigmented mutants. Stud. Mycol. 74, 47-57 (2013). 
14. Kieser, T., Bibb, M.J., Buttner, M.J., Chater, K.F. \& Hopwood, D.A. Practical Streptomyces genetics. Norwich, The John Innes Foundation (2000).

15. Vinck, A. et al. Hyphal differentiation in the exploring mycelium of Aspergillus niger. Mol. Microbiol. 58, $693-699$ (2005).

16. Reichl, U., King, R. \& Gilles, E.D. Characterization of pellet morphology during submerged growth of Streptomyces tendae by image analysis. Biotechnol. Bioeng. 39, 164-170 (1992) 